\title{
Competition between local potentials and attractive particle-particle interactions in superlattices
}

\author{
N. A. Lima ${ }^{a}$ A. L. Malvezzi ${ }^{b}$ K. Capelle ${ }^{c}$ \\ ${ }^{a}$ Colegiado de Engenharia de Produção, Fundação Universidade Federal do Vale \\ do São Francisco, Caixa Postal 252, 56306-410 Petrolina, PE, Brazil \\ ${ }^{\mathrm{b}}$ Departamento de Física, Faculdade de Ciências, Universidade Estadual Paulista, \\ Caixa Postal 473, 17015-970 Bauru, SP, Brazil \\ ${ }^{\mathrm{c}}$ Departamento de Física e Informática, Instituto de Física de São Carlos, \\ Universidade de São Paulo, Caixa Postal 369, 13560-970 São Carlos, SP, Brazil
}

\begin{abstract}
Naturally occuring or man-made systems displaying periodic spatial modulations of their properties on a nanoscale constitute superlattices. Such modulated structures are important both as prototypes of simple nanotechnological devices and as particular examples of emerging spatial inhomogeneity in interacting many-electron systems. Here we investigate the effect different types of modulation of the system parameters have on the ground-state energy and the charge-density distribution of the system. The superlattices are described by the inhomogeneous attractive Hubbard model, and the calculations are performed by density-functional and densitymatrix renormalization group techniques. We find that modulations in local electric potentials are much more effective in shaping the system's properties than modulations in the attractive on-site interaction. This is the same conclusions we previously (Phys. Rev. B 71, 125130) obtained for repulsive interactions, suggesting that it is not an artifact of a specific state, but a general property of modulated structures.
\end{abstract}

Key words: superlattice, electron correlation, nanoscale spatial inhomogeneity, density-functional theory, density-matrix renormalization group

PACS: 71.10.Fd, 71.15.Mb, 71.10.Pm

Email address: capelle@ifsc.usp.br (K. Capelle).

$U R L:$ http://www.ifsc.usp.br/ ${ }^{\sim} \operatorname{dft}($ K. Capelle).

Preprint submitted to Elsevier

25 September 2018 


\section{Introduction}

Superlattices are characterized by a periodic modulation of system properties on a length scale that is larger than the lattice constant of the material they are made of. Superlattices are of great technological interest because the modulation can be used to design the system's optical, electrical, magnetic and transport properties [1,2,3,4,5]. Since the modulation typically takes place on a length scale of nanometers, superlattices also provide a particular realization of systems with nanoscale spatial inhomogeneity, which occurs spontaneously in many strongly correlated systems [6,7,8].

A simple, but nontrivial, model of a superlattice is the inhomogeneous onedimensional Hubbard model (1DHM) for spin 1/2 fermions on a lattice, with Hamiltonian

$$
\hat{H}_{\text {inhom }}=-t \sum_{i, \sigma}\left(c_{i \sigma}^{\dagger} c_{i+1, \sigma}+\text { H.c. }\right)+\sum_{i} U_{i} c_{i \uparrow}^{\dagger} c_{i \uparrow} c_{i \downarrow}^{\dagger} c_{i \downarrow}+\sum_{i \sigma} v_{i} c_{i \sigma}^{\dagger} c_{i \sigma}
$$

where either the on-site interaction $U_{i}$, or the on-site potential $v_{i}$ or both are periodically modulated. Both for designing artificial superlattices and for the study of spontaneously emerging periodic structures, it is important to know which of these two types of modulations is more effective in shaping the system properties.

In Ref. [9] we investigated this question for repulsive interactions, and found that the properties of the superlattice are much more sensitive to local changes in the electric potential than to local changes in the strength of the interparticle interaction. This is a surprising result, as intuitively one would expect that in a strongly correlated system, such as described by the 1DHM, interaction effects should dominate. Indeed, in most applications of the Hubbard model (not only to superlattices, but also, e.g., to HTSC cuprates) the on-site potential is not even considered, or simply assumed to be spatially constant and absorbed in the chemical potential. In the present paper we extend this investigation to attractive interactions, i.e., $U<0$. The negative $U$ Hubbard model has been used to describe superconductivity of strongly correlated electrons, but the same model also describes attractive interactions between cold atoms in optical traps [10].

\section{Comparison of modulated structures}

Many different modulation patterns exist, and many different ways to compare them are conceivable. What we require for our present purposes are quanti- 
tative criteria measuring the relative impact of modulations in the on-site potential, as compared to modulations in the on-site interaction.

In Ref. [9] we introduced two such criteria, which are both based on separate calculations for a 1DHM with no modulations (a spatially uniform system) and for a 1DHM in which $U_{i}$ and $v_{i}$ are modulated with same periodicity but different amplitudes. In the present paper, dedicated to attractive interactions, we choose a periodically repeated pattern of $L_{U}$ sites with interaction $U_{i}<0$ and potential $v_{i}$, followed by $L_{0}$ sites with $U_{i}=v_{i}=0$. The modulations of $U_{i}$ and of $v_{i}$ have distinct effects on the system properties. The ratio of the modulation amplitudes at which the two effects cancel each other, returning effectively to the properties of the uniform system, provides a quantitative measure of their relative impact on the system properties.

Two important ground-state properties of a modulated structure are its total energy and its density profile. These quantities form the basis for the two criteria we employ. In the energy criterium, we vary the on-site potential until the doubly modulated lattice has the same ground-state energy found in the uniform system. The density criterium consists in finding that potential that produces the same density profile obtained in the open uniform system. Exact cancellations are harder to obtain for the density than for the energy, but cancellations to within the error bars of our numerical methods are all that is required for assessing the relative impact of modulating $v_{i}$ and $U_{i}$.

We stress that we do not expect such cancellations to occur frequently in nature. We simply use them here as a convenient way to quantify the relative impact of both types of modulation. Essentially, we are simulating numerically the sensitivity of the energy and the density to changes in the interaction and the potential, i.e., sampling certain response functions.

\section{Computational methods}

We calculate the energies and densities by means of two independently developed and implemented numerical techniques: the density-matrix renormalization group (DMRG) [11,12] and density-functional theory (DFT) within the Bethe-Ansatz local-density approximation (BA-LDA) [14,15,16,17]. Both methods provide energies and density profiles of the Hamiltonian (1), but are complementary in scope. DMRG provides high-precision results, but at considerable computational expense, in particular for systems with reduced spatial symmetries or periodic boundary conditions. BA-LDA easily handles systems of almost arbitrary size and its performance does not significantly depend on presence or absence of spatial symmetries or choice of boundary conditions. Its intrinsic accuracy is, however, limited to a few percent (with 
Table 1

Ground-state energy of superlattices with open boundary conditions, $L$ sites and $N$ electrons, subjected to spatially modulated on-site interaction $U_{i}$ and spatially constant on-site potential $v_{i}=0$, obtained with DMRG and with DFT/BA-LDA. The modulation pattern alternates $L_{U}$ sites of interaction $U<0$ with $L_{0}$ sites with interaction $U=0$, simulating a superlattice composed of alternating superconducting and normal metals. The last column is the deviation of the BA-LDA energies from the DMRG ones (in percent).

\begin{tabular}{c|c|c|c|c|c|c|c}
$L$ & $N$ & $\mathrm{U}$ & $L_{U}$ & $L_{0}$ & $E_{0}^{\mathrm{DMRG}} / t$ & $E_{0}^{\mathrm{BA}-\mathrm{LDA}} / t$ & $\Delta \%$ \\
\hline 60 & 30 & -3 & 60 & 0 & -72.989 & -74.118 & 1.55 \\
60 & 30 & -3 & 3 & 2 & -69.473 & -70.379 & 1.30 \\
100 & 40 & -3 & 1 & 1 & -90.059 & -90.740 & 0.76 \\
100 & 40 & -6 & 1 & 1 & -134.25 & -138.08 & 2.85 \\
300 & 150 & -3 & 10 & 10 & -337.92 & -340.88 & 0.88 \\
300 & 150 & -6 & 10 & 10 & -515.18 & -532.79 & 3.42 \\
300 & 300 & -3 & 10 & 10 & -556.07 & -560.41 & 0.78
\end{tabular}

energies typically being slightly more accurate than densities) and it is not easy to consistently improve on these values.

Within density-functional theory, the ground-state energy and density distribution are obtained by self-consistent solution of a set of single-particle equations, the Kohn-Sham equations, in the presence of effective potentials constructed such as to recover the density distribution of the interacting system. The application of DFT to the Hubbard model was initiated in Refs. [13. Recent progress is due to the development of a simple and accurate Bethe-Ansatz based parametrization for the model's correlation energy in the uniform limit, which can be used to construct a local-density approximation (LDA) [14,15,16]. This parametrization was further developed into a fully numerical scheme [17], which has been applied both to repulsive [17] and attractive [10] interactions, in the context of ultracold fermionic atoms confined in optical traps. In the same context, a simple analytical parametrization of the LDA for attractive interaction was also proposed [18], but applied only in conjunction with a similar LDA for the kinetic energy, leading to a ThomasFermi-like scheme [18].

Here we employ the parametrization for the correlation energy used in Ref. [18], in conjunction with the Kohn-Sham approach of Refs. [14]15]16], to obtain many-body energies and densities. In these calculations a local-density approximation is made only for the correlation energy. Previous work [10], as well as our present results, indicate that the LDA (in either its fully numerical or parametrized forms) for attractive interactions continues to produce energies within a few percent of more precise DMRG data. This is illustrated in 


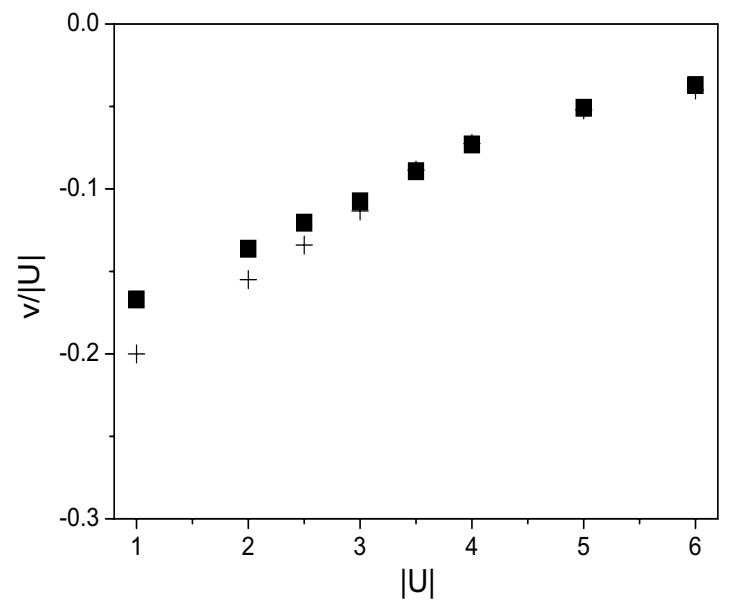

Fig. 1. Amplitude $v$ of the modulation of the on-site potential that produces the same ground-state energy in a superlattice whose interaction is already modulated with amplitude $U$, as obtained in an unmodulated system with interaction $U$ on all sites and $v_{\text {unif }} \equiv 0$. Full squares: DMRG data. Plus: DFT/BA-LDA data. System parameters: $L=60$ sites, $N=30$ fermions, $L_{U}=4$ interacting sites, alternating with $L_{0}=6$ noninteracting sites.

Table 1. DMRG densities are less well reproduced by LDA densities at attractive than at repulsive interactions, but Figs. 1 and 2 show that the differences are far too small to invalidate our conclusions regarding the effect of different modulation patterns.

In view of the complementary aspects of DFT and BA-LDA, we have, both in Ref. [9] and in the present paper, used BA-LDA to efficiently explore large regions of parameter space. In separate calculations we have then used DMRG to fine-tune parameters in the vicinity of the values provided by BA-LDA, and to confirm results with more significant digits. The DMRG calculations were performed using the finite system algorithm under open boundary conditions [11] with truncation errors kept of the order of $10^{-6}$ or smaller.

\section{Attractive Hubbard model: results}

Figure 1 displays representative results from the energy criterium. In these calculations we have chosen a fixed modulation of the interaction, alternating 'layers' of $L_{U}=4$ sites with $U_{i}=U$ and 'layers' of $L_{0}=6$ sites with $U_{i}=0$, and changed the amplitude of the modulation of $v_{i}$ until the doubly modulated system has the same energy as the unmodulated system (with interaction $U$ on all sites and $v_{\text {unif }} \equiv 0$ ). If a different constant value of $v_{\text {unif }}$ is chosen in the uniform system, the compensating values of $v_{i}$ in the doubly modulated 


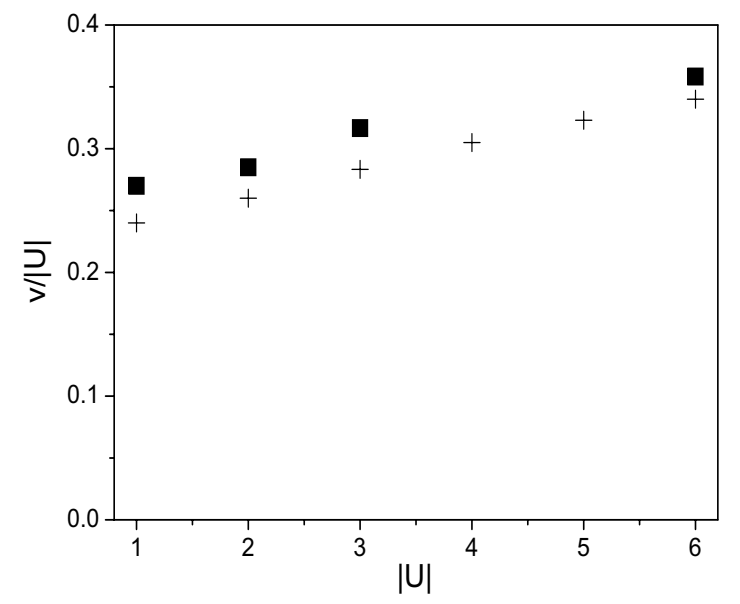

Fig. 2. Amplitude $v$ of the modulation of the on-site potential that produces the same ground-state density distribution in a superlattice whose interaction is already modulated with amplitude $U$, as obtained in an unmodulated system with interaction $U$ on all sites and $v_{\text {unif }} \equiv 0$. Full squares: DMRG data. Plus: DFT/BA-LDA data. System parameters: $L=160$ sites, $N=80$ fermions, $L_{U}=6$ interacting sites, alternating with $L_{0}=10$ noninteracting sites.

system are simply shifted up by that value of $v_{\text {unif }}$, without changing our conclusions.

Figure 1 shows that for physically realistic values of $U$ the effect of a modulation of $U$ on the ground-state energy is reproduced by a modulation of $v$ that is between $10^{-2}$ and $2 \times 10^{-1}$ times smaller. Clearly, the ground-state energy depends much more sensitively on modulations of the on-site potential than on modulations of the on-site interaction. Qualitatively, this is the same conclusion obtained previously for repulsive interactions [9]. Quantitatively, the effect of attractive interactions is even weaker than that of repulsive interactions, as can be seen by comparing the present Fig. 1 with the upper panel of Fig. 4 of Ref. [9]: an even weaker modulation of the potential is sufficient to change the energy by the same margin as a modulation of the interaction.

In Fig. 2 we turn to the density criterium, i.e., investigate for what amplitude of modulation of an repulsive on-site potential $v_{i}$ it cancels the effect of a modulation of the attractive on-site interaction $U_{i}$ on the density profile. The density is more sensitive to small variations in the system parameters, and in general it is more difficult to satisfy the density criterium than the energy criterium. In practice, we have performed a large number of BA-LDA calculations within DFT, until finding values of $v_{i}$ that produce approximately the same density profile. In the vicinity of these BA-LDA values we have then performed a few DMRG calculations, for selected values of $U$, to confirm the observed trend. This procedure is motivated by the large difference in comput- 
ing time required by both methods (seconds to minutes, as compared to hours or days, depending on the system parameters and the required precision).

The data in Fig. 2 show the same trend as those in Fig. 1, indicating that the density profile of the superlattice is about one order of magnitude more sensitive to changes in the potential than in the interaction, even for quite large values of $U$. This is the same finding obtained in Ref. [9] for repulsive interactions. Since the nature of the ground state at attractive and repulsive interactions is very different, we infer that we are observing a general feature of periodically modulated systems, not a specific consequence of one type of ground state. The ratio between compensating potentials and interactions is, however, not the same for the energy criterium and for the density criterium, and also differs, for each criterium, for attractive and for repulsive interactions, which indicates that there is no universal energy scale associated with the compensation.

\section{Conclusions}

Density-functional and density-matrix renormalization group calculations have been performed for superlattices with different modulation patterns, in the presence of attractive interactions. Results for ground-state energies and chargedensity profiles, obtained for different lattices and with DFT or DMRG, are mutually consistent and all point towards the same conclusion: modulations in the on-site potential are much more efficient in shaping the system properties than modulations in the on-site interaction. This observation has two distinct consequences, depending on whether ones interest lies in the design of materials and devices, or in the understanding of spontaneously emerging spatial inhomogeneity.

If one wants to design a superlattice with specific electronic properties, e.g., as part of a nanoscale device, the key parameter to modulate is the local electric potential. Spatial variations of the effective particle-particle interactions are of secondary importance. If, on the other hand, one is given a system in which nanoscale spatial inhomogeneity appears spontaneously, such as in many HTSC cuprates, our results imply that the analysis of origins and consequences of the modulation in terms of strong-correlation (large $U$ ) effects alone is incomplete, and must necesssarily be accompanied by accounting for local electric fields.

\section{Acknowledgments}

This work was supported by FAPESP and CNPq. 


\section{References}

[1] A. L. Malvezzi, T. Paiva, and R. R. dos Santos, Phys. Rev. B 66, 064430 (2002) [Virtual Journal of Nanoscale Science $\mathcal{E}$ Technology 6 (2002) (www.vinano.org)] and Phys. Rev. B 73, 193407 (2006).

[2] J. Silva-Valencia, E. Miranda, and R. R. dos Santos, J. Phys. Condens. Mat. 13, L619 (2001).

[3] T. Paiva and R. R. dos Santos, Phys. Rev. B 65, 153101 (2002), ibid 62, 7007 (2000), ibid 58, 9607 (1998).

[4] D. Góra, K. J. Rościszewski and A. M. Oleś, J. Phys. Condens. Matter 10, 4755 (1998).

[5] M. Noh, D. C. Johnson and G. S. Elliott, Chem. Mater. 12, 2894 (2000).

[6] 'Intrinsic Multiscale Structure and Dynamics in Complex Electronic Oxides', A. R. Bishop, S. R. Shenoy and S. Sridhar, eds. (World Scientific, New Jersey, 2003).

[7] E. W. Hudson, S. H. Pan, A. K. Gupta, K.-W. Ng, and J. C. Davis, Science 285, 88 (1999).

[8] K. M. Lang, V. Madhavan, J. E. Hoffman, E. W. Hudson, H. Eisaki, S. Uchida, and J. C. Davis, Nature 415, 412 (2002).

[9] M. F. Silva, N. A. Lima, A. L. Malvezzi and K. Capelle, Phys. Rev. B 71, 125130 (2005).

[10] G. Xianlong, M. Rizzi, M. Polini, R. Fazio, M. P. Tosi, V. L. Campo Jr. and K. Capelle, cond-mat/0609346.

[11] S. R. White, Phys. Rev. Lett. 69, 2863 (1992), ibid 77, 3633 (1993). Phys. Rev. B 48, 10345 (1993).

[12] A. L. Malvezzi, Braz. J. Phys. 33, 55 (2003).

[13] O. Gunnarsson and K. Schönhammer, Phys. Rev. Lett. 56, 1968 (1986).

[14] N. A. Lima, M. F. Silva, L. N. Oliveira, and K. Capelle, Phys. Rev. Lett. 90, $146402(2003)$.

[15] K. Capelle, N. A. Lima, M. F. Silva, and L. N. Oliveira, in 'The fundamentals of density matrix and density functional theory in atoms, molecules, and solids', N. Gidopoulos and S. Wilson eds. (Kluwer, Dordrecht, 2003) cond-mat/0209245.

[16] N. A. Lima, L. N. Oliveira and K. Capelle, Europhys. Lett. 60, 601 (2002).

[17] G. Xianlong, M. Polini, M. P. Tosi, V. L. Campo, Jr., K. Capelle and M. Rigol Phys. Rev. B 73, 165120 (2006).

[18] V. L. Campo, Jr. and K. Capelle, Phys. Rev. A 72, 061602 (2005). 